TRANSACTIONS OF THE

AMERICAN MATHEMATICAL SOCIETY

Volume 363, Number 9, September 2011, Pages 4585-4601

S 0002-9947(2011)05178-0

Article electronically published on April 11, 2011

\title{
IF YOU CAN HIDE BEHIND IT, CAN YOU HIDE INSIDE IT?
}

\author{
DANIEL A. KLAIN
}

\begin{abstract}
Let $K$ and $L$ be compact convex sets in $\mathbb{R}^{n}$. Suppose that, for a given dimension $1 \leq d \leq n-1$, every $d$-dimensional orthogonal projection of $L$ contains a translate of the corresponding projection of $K$. Does it follow that the original set $L$ contains a translate of $K$ ? In other words, if $K$ can be translated to "hide behind" $L$ from any perspective, does it follow that $K$ can "hide inside" $L$ ?

A compact convex set $L$ is defined to be $d$-decomposable if $L$ is a direct Minkowski sum (affine Cartesian product) of two or more convex bodies each of dimension at most $d$. A compact convex set $L$ is called $d$-reliable if, whenever each $d$-dimensional orthogonal projection of $L$ contains a translate of the corresponding $d$-dimensional projection of $K$, it must follow that $L$ contains a translate of $K$.

It is shown that, for $1 \leq d \leq n-1$ :

(1) $d$-decomposability implies $d$-reliability.

(2) A compact convex set $L$ in $\mathbb{R}^{n}$ is $d$-reliable if and only if, for all $m \geq d+2$, no $m$ unit normals to regular boundary points of $L$ form the outer unit normals of an $(m-1)$-dimensional simplex.

(3) Smooth convex bodies are not $d$-reliable.

(4) A compact convex set $L$ in $\mathbb{R}^{n}$ is 1-reliable if and only if $L$ is 1-decomposable (i.e. a parallelotope).

(5) A centrally symmetric compact convex set $L$ in $\mathbb{R}^{n}$ is 2-reliable if and only if $L$ is 2 -decomposable.

However, there are non-centered 2-reliable convex bodies that are not 2-decomposable.

As a result of (5) above, the only reliable centrally symmetric covers in $\mathbb{R}^{3}$ from the perspective of 2-dimensional shadows are the affine convex cylinders (prisms). However, in dimensions greater than 3, it is shown that 3decomposability is only sufficient, and not necessary, for $L$ to cover reliably with respect to 3 -shadows, even when $L$ is assumed to be centrally symmetric.
\end{abstract}

Consider two compact convex subsets $K$ and $L$ of $n$-dimensional Euclidean space. Suppose that, for a given dimension $1 \leq d<n$, every $d$-dimensional orthogonal projection (shadow) of $L$ contains a translate of the corresponding projection of $K$. Does it follow that the original set $L$ contains a translate of $K$ ? In other words, if $K$ can be translated to "hide behind" $L$ from any perspective, does it follow that $K$ can "hide inside" $L$ ?

In dimension 2 it is easy to see that the answer is no. For example, if an equilateral triangle $\Delta$ is inscribed in a disc $D$ of unit diameter, the slightly larger triangle $(1+\epsilon) \Delta$ still has less than unit width in every direction (provided $\epsilon>0$ is sufficiently small), but no longer fits inside $D$. The same construction works for any set $K$ inscribed in $D$ and having strictly less than unit diameter. Another

Received by the editors June 4, 2009.

2000 Mathematics Subject Classification. Primary 52A20. 
counterexample arises from comparing $\Delta$ and the dilated and reflected triangle $-(1+\epsilon) \Delta$ for small $\epsilon>0$.

Although the details are less obvious, counterexamples also exist in higher dimensions. Let $B$ denote the Euclidean 3-ball of unit radius, and let $T$ denote the regular tetrahedron having edge length $\sqrt{3}$. Jung's Theorem [3, p. 84], 31, p. 320] implies that every 2-projection of $T$ is covered by a translate of the unit disk. But a simple computation shows that $B$ cannot cover the tetrahedron $T$. An analogous construction yields a similar result for higher dimensional simplices and Euclidean balls. One might say that, although $T$ can be translated within a fixed distance from $B$ (i.e. without moving far away) to hide behind $B$ from any observer's perspective, this does not imply that $T$ can hide inside $B$.

Indeed, for $1 \leq d \leq n-1$, it is shown in [17] that, if $K$ is a compact convex set in $\mathbb{R}^{n}$ having at least $d+2$ exposed points, then there exists another compact convex set $L$ such that every $d$-dimensional orthogonal projection (shadow) of $L$ contains a translate of the corresponding projection of $K$, while $L$ does not contain a translate of $K$. In certain cases one can even find examples where $K$ also has larger volume than $L$ (and so certainly could not fit inside $L$ ). For a detailed example of this volume phenomenon, see [16].

This leads to the question: under what additional conditions on either of the sets $K$ or $L$ does covering of the shadows of $K$ by translates of the shadows of $L$ imply covering of the original set $K$ by a translate of the set $L$ ?

This question is easily answered when a sufficient degree of symmetry is imposed. For example, a support function argument implies that shadow covering implies actual covering if both of the bodies $K$ and $L$ are centrally symmetric. It is also not difficult to show that, if every $d$-projection of $K$ (for some $1 \leq d<n$ ) can be translated inside the corresponding shadow of an orthogonal $n$-dimensional box $C$, then $C$ contains a translate of $K$, since one needs only to check that the widths are compatible in the $n$ edge directions of $C$. Related special cases occur if $C$ is a parallelotope (an affine image of a box), or even a cylinder (the product of an $(n-1)$-dimensional compact convex set with a line segment).

In 22 Lutwak uses Helly's theorem to prove that, if every $n$-simplex containing $L$ also contains a translate of $K$, then $L$ contains a translate of $K$. In the present article we generalize Lutwak's theorem in order to reduce questions about shadow covering to questions about circumscribing simplices and simplicial cylinders. A compact convex set $L$ will be called $d$-decomposable if $L$ is a direct Minkowski sum (affine Cartesian product) of two or more convex bodies each of dimension at most $d$ (see Section 11). A compact convex set $L$ will be called $d$-reliable if, whenever each $d$-shadow of $L$ contains a translate of the corresponding $d$-shadow of $K$, it follows that $L$ contains a translate of $K$ (see Section 3). It will be shown that, for $1 \leq d \leq n-1$ :

(1) $d$-decomposability implies $d$-reliability. (Theorem 1.3 ,

(2) A compact convex set $L$ in $\mathbb{R}^{n}$ is $d$-reliable if and only if, for all $m \geq d+2$, no $m$ unit normals to regular boundary points of $L$ form the outer unit normals of an $(m-1)$-dimensional simplex. (Theorem 3.4)

(3) Smooth convex bodies are not $d$-reliable. (Corollary 3.6)

(4) A compact convex set $L$ is 1-reliable if and only if $L$ is 1-decomposable (i.e. a parallelotope). (Corollary 3.7) 
(5) A centrally symmetric compact convex set $L$ is 2-reliable if and only if $L$ is 2-decomposable (Theorem 4.1). However, there are non-centered 2-reliable convex bodies that are not 2-decomposable. (Corollary 3.8)

As a result of (5) above, the only reliable centrally symmetric covers in $\mathbb{R}^{3}$ from the perspective of 2-dimensional shadows are the affine convex cylinders (prisms). However, in dimensions greater than 3 , it will be seen (at the end of Section 4) that 3 -decomposability is only sufficient, and not necessary, for $L$ to cover reliably with respect to 3 -shadows, even when $L$ is assumed to be centrally symmetric.

The containment and covering problems addressed in this article are special cases of the following question: under what conditions will a compact convex set necessarily contain a translate or otherwise congruent copy of another? Progress on different aspects of this general question also appears in the work of Gardner and Volčič [10, Groemer [12, Hadwiger [13, 14, 15, 18, 26, Jung 3, 31, Lutwak [22, Rogers [25], Soltan [30, Steinhagen [3, p. 86], Zhou [34, 35], and others (see also [8]).

These questions are also motivated in part by dramatic progress on two notorious related problems: the Shephard Problem [29] and the Busemann-Petty Problem [5]. Both address properties of bodies $K$ and $L$ that are assumed to be centrally symmetric about the origin (for which these particular questions become non-trivial). The Shephard Problem asks: if each orthogonal $(n-1)$-projection of $K$ has smaller $(n-1)$-volume than the corresponding projection of $L$, does it follow that $K$ has smaller $n$-volume than $L$ ? The Busemann-Petty Problem addresses an analogous question by comparing the volumes of $(n-1)$-dimensional cross-sections of $K$ and $L$ through the origin. The Shephard Problem was solved independently by Petty 24 and Schneider [27, who showed that, while the answer in general is no for dimensions $n \geq 3$, the answer is yes when the convex set $L$ is a projection body; that is, a zonoid. The solution to the Busemann-Petty Problem proved more subtle and elusive. After decades of partial results by several researchers (see, for example, [1, 4, 6, 7, 9, 11, 19, 20, 23, 32, 33]) the surprising answer was revealed to be no for bodies of dimension $n \geq 5$ and yes for bodies of dimension $n \leq 4$. Moreover, in analogy to the Petty-Schneider theorem, the answer is always yes when the set $L$ is an intersection body [21. A more complete discussion of these and related problems (some of which remain open) can be found in the comprehensive book by Gardner [8].

\section{BACKGROUND}

Denote $n$-dimensional Euclidean space by $\mathbb{R}^{n}$, and let $\mathbb{S}^{n-1}$ denote the set of unit vectors in $\mathbb{R}^{n}$; that is, the unit $(n-1)$-sphere centered at the origin.

Denote by $\mathscr{K}_{n}$ the set of compact convex subsets of $\mathbb{R}^{n}$. The $n$-dimensional (Euclidean) volume of a convex set $K$ will be denoted $V_{n}(K)$. If $u$ is a unit vector in $\mathbb{R}^{n}$, denote by $K_{u}$ the orthogonal projection of a set $K$ onto the subspace $u^{\perp}$. More generally, if $\xi$ is a $d$-dimensional subspace of $\mathbb{R}^{n}$, denote by $K_{\xi}$ the orthogonal projection of a set $K$ onto the subspace $\xi$. The boundary of a compact convex set $K$ will be denoted by $\partial K$.

Let $h_{K}: \mathbb{R}^{n} \rightarrow \mathbb{R}$ denote the support function of a compact convex set $K$; that is,

$$
h_{K}(v)=\max _{x \in K} x \cdot v
$$


If $\xi$ is a subspace of $\mathbb{R}^{n}$ then the support function $h_{K_{\xi}}$ is given by the restriction of $h_{K}$ to $\xi$. If $u$ is a unit vector in $\mathbb{R}^{n}$, denote by $K^{u}$ the support set of $K$ in the direction of $u$; that is,

$$
K^{u}=\left\{x \in K \mid x \cdot u=h_{K}(u)\right\} .
$$

If $P$ is a convex polytope, then $P^{u}$ is the face of $P$ having $u$ in its outer normal cone.

Given two $K, L \in \mathscr{K}_{n}$ and $a, b \geq 0$ denote

$$
a K+b L=\{a x+b y \mid x \in K \text { and } y \in L\} .
$$

An expression of this form is called a Minkowski combination or Minkowski sum. Because $K$ and $L$ are convex, the set $a K+b L$ is also convex. Convexity also implies that $a K+b K=(a+b) K$ for all $a, b \geq 0$. Support functions satisfy the identity $h_{a K+b L}=a h_{K}+b h_{L}$. (See, for example, [3, 28, 31.)

If $K \in \mathscr{K}_{n}$ has non-empty interior, define the surface area measure $S_{K}$ on the $(n-1)$-dimensional unit sphere $\mathbb{S}^{n-1}$ as follows: for $A \subseteq \mathbb{S}^{n-1}$ denote by $K^{A}=\bigcup_{u \in A} K^{u}$, and define $S_{K}(A)=\mathcal{H}_{n-1}\left(K^{A}\right)$, the $(n-1)$-dimensional Hausdorff measure of the subset $K^{A}$ of the boundary of $K$. (See [28, p. 203].) If $P$ is a polytope, then $S_{P}$ is a pointed measure concentrated at precisely those directions $u$ that are outer normals to the facets of $P$.

The measure $S_{K}$ is easily shown to satisfy the property

$$
\int_{\mathbb{S}^{n-1}} u d S_{K}=\vec{o},
$$

that is, the mass distribution on the sphere described by $S_{K}$ has center of mass at the origin. For a convex polytope $P$ having outward facet unit normals $u_{1}, \ldots, u_{m}$ and corresponding facet areas $\alpha_{1}, \ldots, \alpha_{m}>0$, the identity (0.1) takes the simple and intuitive form:

$$
\alpha_{1} u_{1}+\cdots+\alpha_{m} u_{m}=\vec{o} .
$$

Minkowski's Existence Theorem [3. p. 125], [28, p. 390] gives a useful converse to the identity (0.1): if $\mu$ is a non-negative measure on the unit sphere $\mathbb{S}^{n-1}$ such that $\mu$ has center of mass at the origin, and if $\mu$ is not concentrated on any great (equatorial) $(n-1)$-subsphere, then $\mu=S_{K}$ for some $K \in \mathscr{K}_{n}$. Moreover, this convex body $K$ is unique up to translation.

Suppose that $\mathscr{F}$ is a family of compact convex sets in $\mathbb{R}^{n}$. Helly's theorem 3. 28, 31] asserts that if every $n+1$ sets in $\mathscr{F}$ share a common point, then the entire family shares a common point. In 22] Lutwak used Helly's theorem to prove the following fundamental criterion for whether a set $L \in \mathscr{K}_{n}$ contains a translate of another set $K \in \mathscr{K}_{n}$.

Theorem 0.1 (Lutwak's containment theorem). Let $K, L \in \mathscr{K}^{n}$. The following are equivalent:

(i) For every simplex $\Delta$ such that $L \subseteq \Delta$, there exists $v \in \mathbb{R}^{n}$ such that $K+v \subseteq$ $\Delta$.

(ii) There exists $v_{0} \in \mathbb{R}^{n}$ such that $K+v_{0} \subseteq L$.

In other words, if every $n$-simplex containing $L$ also contains a translate of $K$, then $L$ contains a translate of $K$. 


\section{Circumscribing Sets And shadows}

A convex set $K \in \mathscr{K}_{n}$ will be called $d$-decomposable if there exists a subspace decomposition

$$
\mathbb{R}^{n}=\xi_{1} \oplus \cdots \oplus \xi_{m},
$$

where $\operatorname{dim} \xi_{i} \leq d$ for each $i$, and compact convex sets $K_{i} \subseteq \xi_{i}$ for each $i$, such that $K=K_{1}+\cdots+K_{m}$. Decompositions of this kind will be denoted

$$
K=K_{1} \oplus \cdots \oplus K_{m} .
$$

If $K=\Delta_{1} \oplus \cdots \oplus \Delta_{m}$, where the component sets $\Delta_{i}$ are simplices, each of dimension at most $d$, then we will say that $K$ is a $d$-decomposable simplex product. The product will be called orthogonal if the subspaces $\xi_{i}$ are mutually orthogonal.

The 2-decomposable sets in $\mathbb{R}^{3}$ (as well as products of $(n-1$ )-dimensional sets with line segments in $\mathbb{R}^{n}$ ) are often called cylinders or prisms.

If the circumscribing simplices are replaced by circumscribing simplex products for $L$, then the following generalization of Lutwak's theorem 0.1 is obtained.

Theorem 1.1 (Prismatic containment theorem). Let $K, L \in \mathscr{K}^{n}$. The following are equivalent:

(i) For every d-decomposable set $C \in \mathscr{K}_{n}$ such that $L \subseteq C$, there exists $v \in \mathbb{R}^{n}$ such that $K+v \subseteq C$.

(ii) For every d-decomposable orthogonal simplex product $C$ such that $L \subseteq C$, there exists $v \in \mathbb{R}^{n}$ such that $K+v \subseteq C$.

(iii) For every d-dimensional subspace $\xi \subseteq \mathbb{R}^{n}$, there exists $w \in \xi$ such that $K_{\xi}+w \subseteq L_{\xi}$.

In other words, if every $d$-decomposable (simplex) product $C$ containing $L$ also contains a translate of $K$, then every $d$-shadow $L_{\xi}$ contains a translate of the corresponding shadow $K_{\xi}$, and vice versa.

The following proposition will simplify the proof of Theorem 1.1 .

Proposition 1.2. Let $K, L \in \mathscr{K}_{n}$. Let $\psi: \mathbb{R}^{n} \rightarrow \mathbb{R}^{n}$ be a non-singular linear transformation. Then $L_{u}$ contains a translate of $K_{u}$ for all unit directions $u$ if and only if $(\psi L)_{u}$ contains a translate of $(\psi K)_{u}$ for all $u$.

This proposition implies that nothing is gained (or lost) by allowing more general (possibly non-orthogonal) linear projections.

Proof. For $S \subseteq \mathbb{R}^{n}$ and a non-zero vector $u$, let $\mathcal{L}_{S}(u)$ denote the set of straight lines in $\mathbb{R}^{n}$ parallel to $u$ and meeting the set $S$. The projection $L_{u}$ contains a translate of $K_{u}$ for each unit vector $u$ if and only if, for each $u$, there exists $v_{u}$ such that

$$
\mathcal{L}_{K+v_{u}}(u) \subseteq \mathcal{L}_{L}(u) .
$$

But $\mathcal{L}_{K+v_{u}}(u)=\mathcal{L}_{K}(u)+v_{u}$ and $\psi \mathcal{L}_{K}(u)=\mathcal{L}_{\psi K}(\psi u)$. It follows that (1.1) holds if and only if $\mathcal{L}_{K}(u)+v_{u} \subseteq \mathcal{L}_{L}(u)$, if and only if

$$
\mathcal{L}_{\psi K}(\psi u)+\psi v_{u} \subseteq \mathcal{L}_{\psi L}(\psi u) \text { for all unit } u .
$$

Set

$$
\tilde{u}=\frac{\psi u}{|\psi u|} \quad \text { and } \quad \tilde{v}=\psi v_{u}
$$


The relation (1.1) now holds if and only if, for all $\tilde{u}$, there exists $\tilde{v}$ such that

$$
\mathcal{L}_{\psi K}(\tilde{u})+\tilde{v} \subseteq \mathcal{L}_{\psi L}(\tilde{u}),
$$

which holds if and only if $(\psi L)_{\tilde{u}}$ contains a translate of $(\psi K)_{\tilde{u}}$ for all $\tilde{u}$.

Proof of Theorem 1.1. To begin, note that (i) implies (ii) trivially.

Suppose that (ii) holds. Given a $d$-subspace $\xi \subseteq \mathbb{R}^{n}$, let $T$ be a simplex in $\xi$ that circumscribes $L_{\xi}$. Let $u_{d+1}, \ldots, u_{n}$ be an orthonormal basis for $\xi^{\perp}$, and let $C^{\prime}$ be a cube in $\xi^{\perp}$ with edges parallel to the directions $u_{i}$ and large enough so that $L_{\xi^{\perp}} \subseteq C^{\prime}$. Now let $C=T \oplus C^{\prime}$. Since $L \subseteq C$, it follows from (ii) that there exists $v \in \mathbb{R}^{n}$ such that $K+v \subseteq C$. This implies that $K_{\xi}+v_{\xi} \subseteq C_{\xi}=T$. On applying Lutwak's theorem 0.1 in the subspace $\xi$ it follows that $K_{\xi}+w \subseteq L_{\xi}$ for some $w \in \xi$. Therefore, (ii) implies (iii).

Next, suppose that (iii) holds. If $L \subseteq C=C_{1} \oplus \cdots \oplus C_{m}$, where each $\operatorname{dim} \xi_{i} \leq d$, then let $\psi$ be a non-singular linear operator on $\mathbb{R}^{n}$ such that the subspaces $\psi\left(\xi_{i}\right)$ are mutually orthogonal. By Proposition 1.2 the condition (iii) also holds for $\psi K$ and $\psi L$. For each $i$ we obtain $v_{i} \in \psi \xi_{i}$ such that

$$
(\psi K)_{\psi \xi_{i}}+v_{i} \subseteq(\psi L)_{\psi \xi_{i}} \subseteq(\psi C)_{\psi \xi_{i}}=\psi C_{i} .
$$

Let $v=v_{1}+\cdots+v_{m}$. Since the subspaces $\psi \xi_{i}$ are mutually orthogonal, we have $\psi K+v \subseteq \psi C$, so that $K+\psi^{-1} v \subseteq C$. Therefore, (iii) implies (i), and the three assertions are equivalent.

It is worth noting the following special case of Theorem 1.1

Theorem 1.3. Let $K, C \in \mathscr{K}^{n}$, where $C$ is d-decomposable.

Suppose that, for each $d$-dimensional subspace $\xi \subseteq \mathbb{R}^{n}$, there exists $w \in \xi$ such that $K_{\xi}+w \subseteq C_{\xi}$. Then there exists $v \in \mathbb{R}^{n}$ such that $K+v \subseteq C$.

When $d=n-1$, Theorem 1.3 says that if you can hide behind a cylinder from any perspective (and without rotating), then you can also hide inside the cylinder.

More consequences of Theorem 1.1 are explored in [16].

\section{Simplicial families of UNit NORMALS}

Theorem 1.3 motivates a converse question: if $L$ is not $d$-decomposable, does there necessarily exist $K$ such that every $d$-shadow of $L$ contains a translate of the corresponding $d$-shadow of $K$, while $L$ itself does not contain a translate of $K$ ? The answer is not necessarily. We will show in a later section (see Corollary 3.8) that if $L$ is a square pyramid (the convex hull of a square in $\mathbb{R}^{3}$ with a point above its center), then no $K$ can hide behind $L$ unless $K$ can also hide inside $L$. However, the square pyramid is not 2-decomposable. In other words, the condition of being $d$-decomposable is sufficient, but not necessary.

In this section we develop some tools for constructing necessary and sufficient conditions for when shadow covering implies actual covering. These tools are applied in later sections.

A set of unit vectors $\left\{u_{0}, \ldots, u_{d}\right\} \subseteq \mathbb{S}^{n-1}$ will be called a $d$-simplicial family, or $d$-simplicial, if $u_{0}, \ldots, u_{d}$ span a $d$-dimensional subspace of $\mathbb{R}^{n}$, and if there exist real numbers $c_{0}, \ldots, c_{d}>0$, such that

$$
c_{0} u_{0}+c_{1} u_{1}+\cdots+c_{d} u_{d}=o .
$$


Equivalently, $u_{0}, \ldots, u_{d}$ are the outer unit normals of some $d$-dimensional simplex. Note that a $d$-simplicial family contains exactly $d+1$ unit vectors.

It will be seen in Sections 3 and 4 that certain translative covering properties of a compact convex set $L$ hinge in the existence of simplicial families of unit normals to regular points of $L$. The next three propositions will be used in that context. (Readers in a hurry may wish to scan Sections 3 and 4 and return to these technical points later on.)

Proposition 2.1. Suppose that $A=\left\{u_{1}, \ldots, u_{m}\right\} \subseteq \mathbb{S}^{n-1}$ contains no simplicial families of size 3 or greater, and that

$$
c_{1} u_{1}+\cdots+c_{m} u_{m}=o
$$

for some $c_{1}, \ldots, c_{m}>0$. Then $m=2 s$ for some integer $s$, and there exist linearly independent vectors $v_{1}, \ldots, v_{s} \in \mathbb{S}^{n-1}$, where $s \leq n$, such that

$$
A=\left\{ \pm v_{1}, \ldots, \pm v_{s}\right\} .
$$

Proof. By (2.1) the set $A$ must have at least 2 elements, and if $A$ has size 2 then the proposition is trivial.

Suppose that the proposition fails for some set $A$ of minimal size $m$, where $m>2$. By (2.1) there exists a minimal subfamily $\left\{u_{i_{1}}, \ldots, u_{i_{k}}\right\} \subseteq A$ such that

$$
a_{1} u_{i_{1}}+\cdots+a_{k} u_{i_{k}}=o
$$

for some $a_{1}, \ldots, a_{k}>0$. Let $d=\operatorname{dim}\left(\operatorname{Span}\left\{u_{i_{1}}, \ldots, u_{i_{k}}\right\}\right)$. Since the $u_{i_{j}}$ are dependent, we have $k \geq d+1$. If $k>d+1$ then Carathéodory's theorem [28, p. 3] (applied in the span of the $\left\{u_{i_{j}}\right\}$ ) implies that the origin $o$ lies in the convex hull of a sub-subfamily of size at most $d+1<k$ of the $u_{i_{j}}$, violating the minimality of $k$. Therefore $k=d+1$, and $\left\{u_{i_{1}}, \ldots, u_{i_{k}}\right\}$ is a simplicial set. By the original assumption on simplicial families in $A$, it follows that $k=2$, so that $u_{i}=-u_{j}$ for some $i \neq j$.

Without loss of generality, suppose that $u_{1}=-u_{2}$ and that $c_{1} \geq c_{2}$. It now follows from (2.1) that

$$
o=\left(c_{1}-c_{2}\right) u_{1}+c_{3} u_{3}+\cdots+c_{m} u_{m} .
$$

Suppose $c_{1}-c_{2}>0$. The minimality of $m$ implies that the proposition holds for the set $\left\{u_{1}, u_{3}, \ldots, u_{m}\right\}$, so that $m-1$ is even and these remaining vectors $u_{1}, u_{3}, \ldots, u_{m}$ can be partitioned into distinct antipodal pairs. Since $u_{1}=-u_{2}$, this would violate the original assumption that the $u_{i}$ are distinct. Therefore $c_{1}=c_{2}$, and

$$
o=c_{3} u_{3}+\cdots+c_{m} u_{m} .
$$

Once again the minimality of $m$ implies that the proposition holds for the set $u_{3}, \ldots, u_{m}$, so that $m-2$ is even (and therefore $m$ is even), and the remaining $u_{i}$ can be separated into distinct antipodal pairs $\pm v_{2}, \ldots, \pm v_{s}$, where the $v_{i}$ are linearly independent.

It remains to show that $u_{1}$ (and similarly $u_{2}$ ) is linearly independent from the vectors $v_{i}$. If $u_{1}$ lies in the span of $v_{2}, \ldots, v_{s}$, then $u_{1}$ lies in the span of a minimal subset of $v_{2}, \ldots, v_{k}$ of size at least 2 , since $u_{1}$ is distinct from each $\pm v_{i}$. The resulting linear dependence relation violates the non-existence of simplicial subsets of size 3 or greater inside $A$.

Setting $v_{1}=u_{1}$ now completes the proof of the proposition. 
Proposition 2.2. Suppose that $A \subseteq \mathbb{S}^{n-1}$ contains no simplicial sets of size 3 or greater, and that the origin lies in the interior of the convex hull of $A$. Then there exist linearly independent vectors $v_{1}, \ldots, v_{n} \in \mathbb{S}^{n-1}$, such that

$$
A=\left\{ \pm v_{1}, \ldots, \pm v_{n}\right\} \text {. }
$$

Proof. By Carathéodory's theorem there exists a finite subfamily $u_{1}, \ldots, u_{m}$ of $A$, such that (2.1) holds. Since $o$ lies in the interior of the convex hull of $A$, we can take $m$ large enough so that $u_{1}, \ldots, u_{m}$ spans $\mathbb{R}^{n}$. By Proposition 2.1, this subfamily has the form $\left\{ \pm v_{1}, \ldots, \pm v_{s}\right\} \subseteq A$, where $v_{1}, \ldots, v_{s}$ are linearly independent and span $\mathbb{R}^{n}$. It follows that $s=n$.

If $w \in A$ and $w \neq \pm v_{i}$, then $w$ lies in the span of some $v_{i_{1}}, \ldots, v_{i_{k}}$, where $k \geq 2$ is minimal. This linear dependence relation violates the non-existence of simplicial sets of size 3 or greater inside the set $A$.

It follows that $\left\{ \pm v_{1}, \ldots, \pm v_{n}\right\}=A$.

Proposition 2.3. Suppose that $A \subseteq \mathbb{S}^{n-1}$ is symmetric under reflection through the origin; that is, $A=-A$. Suppose also that $A$ contains no simplicial sets of size 4 or greater, and that the origin lies in the interior of the convex hull of $A$.

Then there exists a subspace direct sum decomposition

$$
\mathbb{R}^{n}=W_{1} \oplus \cdots \oplus W_{k},
$$

where each $\operatorname{dim} W_{i} \leq 2$, and such that $A \subseteq W_{1} \cup \cdots \cup W_{k}$.

Proof. Since $A=-A$, the set $A$ is composed of antipodal pairs $\pm v$ of unit vectors. Moreover, since the convex hull of $A$ has interior and is centrally symmetric, there exist at least $n$ pairs $\pm u_{1}, \ldots, \pm u_{n}$ in $A$ whose $n$ directions are linearly independent. If $A=\left\{ \pm u_{1}, \ldots, \pm u_{n}\right\}$, then $\mathbb{R}^{n}$ is a direct sum of the lines spanned by each $\pm u_{i}$, and the proposition follows.

If, instead, $\pm v$ is another antipodal pair in $A$, where $v \neq \pm u_{i}$ for all $i$, then without loss of generality (relabeling the signs on $\pm u_{i}$ as needed), we have

$$
-v=c_{1} u_{1}+\cdots+c_{d} u_{d}
$$

for some $c_{1}, \ldots, c_{d}>0$, where $d$ is minimal. If $d \geq 3$, then the relation

$$
v+c_{1} u_{1}+\cdots+c_{d} u_{d}=0
$$

implies that $\left\{v, u_{1}, \ldots, u_{d}\right\}$ form a simplicial family in $A$ of size at least 4 , contradicting hypothesis. Meanwhile, since $v \neq \pm u_{i}$, we must have $d>1$. The remaining possibility is $d=2$, so that $v$ lies in the span of $\left\{u_{1}, u_{2}\right\}$.

If $w \in A$ and $w \neq \pm v, \pm u_{1}, \ldots, \pm u_{n}$, then $w$ lies in the span of 2 of the $u_{i}$ by a similar argument. But if $w=a_{1} u_{1}+a_{3} u_{3}$, say, where $a_{1}, a_{3}>0$, then

$$
w=\frac{a_{1}}{c_{1}}\left(-c_{2} u_{2}-v\right)+a_{3} u_{3}=\frac{a_{1} c_{2}}{c_{1}}\left(-u_{2}\right)+\frac{a_{1}}{c_{1}}(-v)+a_{3} u_{3} .
$$

Since every 3 of the 4 vectors $v, w, u_{2}, u_{3}$ are linearly independent, we obtain a simplicial set of size 4, another contradiction. Therefore, either $w$ also lies in the span of $\left\{u_{1}, u_{2}\right\}$ or in the span of $\left\{u_{i}, u_{j}\right\}$ for $j>i>2$. An iteration of this argument implies that $\mathbb{R}^{n}$ is decomposed into a direct sum $\mathbb{R}^{n}=W_{1} \oplus \cdots \oplus W_{\left\lfloor\frac{n+1}{2}\right\rfloor}$ of subspaces $W_{i}$ each having dimension at most 2, and where every $v \in A$ also lies in some $W_{i}$.

We will also need the following proposition, which clears up ambiguities regarding when shadows cover inside a larger ambient space. 
Proposition 2.4. Suppose that $\xi$ is a linear flat in $\mathbb{R}^{n}$. Let $K$ and $L$ be compact convex sets in $\xi$. Suppose that, for each $d$-subspace $\eta \subseteq \xi$, the projection $L_{\eta}$ contains a translate of $K_{\eta}$. Then $L_{\eta}$ contains a translate of $K_{\eta}$ for every $d$-subspace $\eta \subseteq \mathbb{R}^{n}$.

In other words, embedding $K$ and $L$ in a higher-dimensional space does not change whether or not every $d$-shadow of $L$ contains a translate of the corresponding $d$-shadow of $K$ (even though there are now more shadow directions to verify).

Proof. Suppose that $\eta$ is a $d$-subspace of $\mathbb{R}^{n}$. Let $\hat{\eta}$ denote the orthogonal projection of $\eta$ into $\xi$. Since $\operatorname{dim}(\hat{\eta}) \leq \operatorname{dim}(\eta)=d$, we can translate $K$ and $L$ inside $\xi$ so that $K_{\hat{\eta}} \subseteq L_{\hat{\eta}}$. Let us assume this translation has taken place. Note that, for $v \in \hat{\eta}$, we now have $h_{K}(v) \leq h_{L}(v)$.

If $u \in \eta$, then express $u=u_{\xi}+u_{\xi^{\perp}}$. Since $K \subseteq \xi$,

$$
h_{K}(u)=\max _{x \in K} x \cdot u=\max _{x \in K} x \cdot u_{\xi}=h_{K}\left(u_{\xi}\right),
$$

and similarly for $L$. But since $u \in \eta$, we have $u_{\xi} \in \hat{\eta}$, so that

$$
h_{K}(u)=h_{K}\left(u_{\xi}\right) \leq h_{L}\left(u_{\xi}\right)=h_{L}(u) .
$$

In other words, $K_{\eta} \subseteq L_{\eta}$.

\section{When CAN A CONVEX SET CONCEAL Without COVERING?}

We now address the possibility of a converse to Theorem 1.3

Definition 3.1. Suppose that $1 \leq d \leq n-1$. A compact convex set $L$ in $\mathbb{R}^{n}$ is said to be a $d$-reliable cover, or $d$-reliable, if whenever $K \in \mathscr{K}_{n}$ and every $d$-shadow $L_{\xi}$ contains a translate of the corresponding shadow $K_{\xi}$, it follows that $L$ contains a translate of $K$.

Evidently, if $L$ is $d$-reliable, then $L$ is also $m$-reliable for all $m>d$.

Theorem 1.3 asserts that if $L$ is $d$-decomposable, then $L$ is also $d$-reliable. However, we will see that a square pyramid gives a counterexample to the converse assertion. It is 2-reliable, but not 2-decomposable (Corollary 3.8).

The next two theorems describe a necessary and sufficient condition for $L$ to be a $d$-reliable cover. Recall that a point $x$ on the boundary of a compact convex set $L$ is said to be regular if the outward normal cone to $L$ at $x$ contains exactly one unit vector.

Theorem 3.2. Suppose that $L$ has regular boundary points $x_{0}, \ldots, x_{d+1}$, whose corresponding unit normals $u_{0}, \ldots, u_{d+1}$ are a simplicial family. Then there exists a polytope $S$ such that $L_{\xi}$ contains a translate of $S_{\xi}$ for each $d$-subspace $\xi$ of $\mathbb{R}^{n}$, while $L$ does not contain a translate of $S$. In particular, $L$ is not d-reliable.

Proof. First, note that, by Proposition 2.4, it is sufficient to prove this theorem for the case in which $L$ has interior. For if $L$ lacks interior, we simply restrict our attention to the affine hull of $L$. Once the theorem is verified in this case, one can apply Proposition 2.4 to verify the theorem when $L$ is re-embedded in a higher-dimensional space. So let us now assume that $L$ has interior.

Suppose that $L$ has regular boundary points $x_{0}, \ldots, x_{d+1}$, as in the hypothesis of the theorem. Let $S$ be the convex hull of $\left\{x_{0}, \ldots, x_{d+1}\right\}$. Evidently $S \subseteq L$. Since $\left\{u_{0}, \ldots, u_{d+1}\right\}$ is a simplicial family, there exist $c_{i}>0$ such that

$$
c_{0} u_{0}+\cdots+c_{d+1} u_{d+1}=o .
$$


Moreover, every $d+1$ of the $u_{i}$ are linearly independent, so that no subfamily of the $u_{i}$ contains the origin in its convex hull, and the property (3.1) does not hold for any subfamily.

Since $S \subseteq L$, we have $h_{S} \leq h_{L}$. Moreover, by our choices of $x_{i}$ and $u_{i}, h_{L}\left(u_{i}\right)=$ $u_{i} \cdot x_{i} \leq h_{S}\left(u_{i}\right)$. Therefore, $h_{L}\left(u_{i}\right)=h_{S}\left(u_{i}\right)$ for each $i$.

Let $\xi$ be a $d$-dimensional subspace of $\mathbb{R}^{n}$. Let $\pi_{\xi}$ denote the orthogonal projection of $\mathbb{R}^{n}$ onto $\xi$. If $\pi_{\xi}\left(x_{i}\right)$ lies on the boundary of $L_{\xi}$, then $h_{L_{\xi}}(w)=x_{i} \cdot w$ for some unit $w \in \xi$. Since $h_{L_{\xi}}$ is given by the restriction of $h_{L}$ to the subspace $\xi$, it follows that $h_{L}(w)=x_{i} \cdot w$. By the regularity of the boundary point $x_{i}$, we have $w=u_{i}$, so that $u_{i} \in \xi$.

Similarly, if $u_{i} \in \xi$, then

$$
h_{L_{\xi}}\left(u_{i}\right)=h_{L}\left(u_{i}\right)=x_{i} \cdot u_{i}=\pi_{\xi}\left(x_{i}\right) \cdot u_{i},
$$

so that $\pi_{\xi}\left(x_{i}\right)$ lies on the boundary of $L_{\xi}$, with outward unit normal $u_{i}$.

Since the $u_{i}$ form a simplicial family of size $d+2$, at most $d$ of these vectors $u_{i}$ can lie in $\xi$. It follows that $S_{\xi}$ meets the boundary of $L_{\xi}$ at $j+1$ points, for some $j \leq d-1$, having outward normals $u_{0}, \ldots, u_{j}$ (without loss of generality). Since $o$ does not lie in the convex hull of $u_{0}, \ldots, u_{j}$, there exists a unit vector $v \in \xi$ such that $v \cdot u_{i}<0$ for $i \leq j$.

Let $y_{i}=\pi_{\xi}\left(x_{i}\right)$. If $i \leq j$, then $y_{i}$ is a regular point of the boundary $\partial L_{\xi}$ with outward unit normal $u_{i}$. Since each $v \cdot u_{i}<0$ in this case, there exists $\epsilon>0$ such that each $y_{i}+\epsilon v$ lies in the relative interior of $L_{\xi}$.

Meanwhile, if $i>j$, then $y_{i}$ lies in the relative interior of $L_{\xi}$ already, so that $y_{i}+\epsilon v$ lies in the relative interior of $L_{\xi}$ as well, provided we have chosen $\epsilon>0$ small enough. In other words, there exists $\epsilon>0$ so that $y_{i}+\epsilon v$ lies in the relative interior of $L_{\xi}$ for all $i$.

Let $T=S_{\xi}+\epsilon v$. Since the polytope $T$ is the convex hull of the points $y_{i}+\epsilon v$, it follows that $T$ lies in the relative interior of $L_{\xi}$. Therefore, there exists $a_{\xi}>1$ such that $L_{\xi}$ contains a translate of $a_{\xi} T$, whence $L_{\xi}$ contains a translate of $a_{\xi} S_{\xi}$.

Since the set of all $d$-subspaces of $\mathbb{R}^{n}$ is compact, there exists $\alpha>1$, independent of $\xi$, such that some translate of $\alpha S_{\xi}$ lies inside $L_{\xi}$ for each $\xi$.

On the other hand, if $\alpha S+w \subseteq L$ for some $w$, then

$$
h_{L}\left(u_{i}\right) \geq h_{\alpha S+w}\left(u_{i}\right)=\alpha h_{S}\left(u_{i}\right)+u_{i} \cdot w=\alpha h_{L}\left(u_{i}\right)+u_{i} \cdot w>h_{L}\left(u_{i}\right)+u_{i} \cdot w,
$$

so that $u_{i} \cdot w<0$ for all $i$. This strict inequality contradicts (3.1).

To prove the converse to Theorem 3.2, we first consider the polytope case. Recall that a facet of a polytope $Q$ is a face of co-dimension 1 in the affine hull of $Q$.

Theorem 3.3. Let $K \in \mathscr{K}_{n}$, and let $Q$ be a convex polytope in $\mathbb{R}^{n}$. Suppose that $Q_{\xi}$ contains a translate of $K_{\xi}$ for every $d$-subspace $\xi$, and that $Q$ does not contain a translate of $K$. Then there exists a simplicial family of facet unit normals $\left\{u_{0}, \ldots, u_{m}\right\}$ to $Q$, for some $m \geq d+1$.

In other words, if a polytope $Q$ is not $d$-reliable, then $Q$ has a simplicial family of facet unit normals of size at least $d+2$.

Proof. As in the previous proof, Proposition 2.4 makes it sufficient to verify the case in which $Q$ has interior. 
Suppose that $K_{\xi}$ can be translated inside $Q_{\xi}$ for each $d$-subspace $\xi$, while $K$ cannot be translated inside $Q$. Without loss of generality, translate $K$ so that the origin $o$ lies inside the relative interior of $K$. This implies that $h_{K} \geq 0$.

Since $Q$ has interior, there exists $\epsilon>0$ such that $\epsilon K$ can be translated inside $Q$. Since $Q$ is compact we may assume $\epsilon$ to be maximal. Evidently $\epsilon<1$, since no translate of $K$ fits inside $Q$. Without loss of generality, translate $Q$ so that $\epsilon K \subseteq Q$.

Denote the facets of $Q$ by $F_{0}, \ldots, F_{q}$, having outward unit normals $u_{0}, \ldots, u_{q}$. Suppose that $\epsilon K$ meets facets $F_{0}, \ldots, F_{m}$, and misses the others.

If the convex hull of $\left\{u_{0}, \ldots, u_{m}\right\}$ does not contain the origin $o$, then there exists a vector $v$ such that $v \cdot u_{i}<0$ for $i=0, \ldots, m$. This implies that, for sufficiently small $\delta$, the translate $\epsilon K+\delta v$ lies in the interior of $Q$. This violates the maximality of $\epsilon$. Therefore, there exist $a_{0}, \ldots, a_{m} \geq 0$ such that

$$
a_{0} u_{0}+\cdots+a_{m} u_{m}=o .
$$

Renumbering the facets as necessary, we have

$$
c_{0} u_{0}+\cdots+c_{s} u_{s}=o,
$$

where each $c_{i}>0$ and $s$ is minimal, so that $\left\{u_{0}, \ldots, u_{s}\right\}$ is a simplicial family.

If $s \leq d$, then the $s+1$ unit vectors $u_{i}$ lie inside a $d$-subspace $\xi$. Since $\epsilon K$ meets each of the facets $F_{0}, \ldots, F_{s}$, we have

$$
\epsilon h_{K}\left(u_{i}\right)=h_{\epsilon K}\left(u_{i}\right)=h_{Q}\left(u_{i}\right)=h_{Q_{\xi}}\left(u_{i}\right)
$$

for each $i=0, \ldots, s$. Since $Q_{\xi}$ contains a translate of $K_{\xi}$, there exists $w \in \xi$ so that $K_{\xi}+w \subseteq Q_{\xi}$, and

$$
\epsilon h_{K}\left(u_{i}\right)=h_{Q_{\xi}}\left(u_{i}\right) \geq h_{K_{\xi}}\left(u_{i}\right)+w \cdot u_{i}=h_{K}\left(u_{i}\right)+w \cdot u_{i}
$$

for each $i=0, \ldots, s$. After summing over $i$, it follows from (3.2) that

$$
\epsilon \sum_{i=0}^{s} c_{i} h_{K}\left(u_{i}\right) \geq \sum_{i=0}^{s} c_{i} h_{K}\left(u_{i}\right)+w \cdot \sum_{i=0}^{s} c_{i} u_{i}=\sum_{i=0}^{s} c_{i} h_{K}\left(u_{i}\right) .
$$

Recall that $h_{K} \geq 0$ and each $c_{i}>0$. Since $\epsilon<1$, it follows that

$$
\sum_{i=0}^{s} c_{i} h_{K}\left(u_{i}\right)=0
$$

so that each $h_{K}\left(u_{i}\right)=0$. Therefore, each $h_{Q}\left(u_{i}\right)=0$, by (3.3). It now follows from (3.2) and the sublinearity of the support function $h_{Q}$ that the projection of $Q$ onto the span of $\left\{u_{0}, \ldots, u_{s}\right\}$ is a single point. This is a contradiction, since $Q$ has interior. It follows that $s \geq d+1$.

Therefore, there exists a simplicial family of facet unit normals $u_{0}, \ldots, u_{s}$ to $Q$, where $s \geq d+1$.

Putting Theorems 3.2 and 3.3 together, we obtain the following.

Theorem 3.4 (Reliability theorem). Let $L \in \mathscr{K}_{n}$. Then $L$ is a d-reliable cover if and only if every simplicial family of normals to regular boundary points of $L$ has size at most $d+1$.

Proof. Suppose a simplicial family of unit normals to regular boundary points of $L$ has size $d+2$ or greater. By Theorem $3.2, L$ is not $d$-reliable. 
To prove the converse, suppose that $L$ is not $d$-reliable. Then there exists $K \in$ $\mathscr{K}_{n}$ such that $L_{\xi}$ contains a translate of $K_{\xi}$ for every $d$-subspace $\xi$, while $L$ does not contain a translate of $K$.

Since regular points are dense on the boundary of $L$ (see [28, p. 73]), there exists a countable dense set of regular points on the boundary of $L$. By intersecting half-spaces that support $L$ at these points, construct a sequence of polytopes $P_{i}$, decreasing with respect to set inclusion, such that $P_{i} \rightarrow L$ and each $P_{i}$ has facet normals that are unit normals at regular points of $L$.

If $P_{i}$ contains a translate of $K$ for all $i$, then so does $L$, a contradiction. Therefore, there exists $j$ such that $P_{j}$ does not contain a translate of $K$. But each projection $L_{\xi} \subseteq\left(P_{j}\right)_{\xi}$, so that each projection $\left(P_{j}\right)_{\xi}$ contains a translate of $K_{\xi}$. In other words, the polytope $P_{j}$ is not $d$-reliable. By Theorem 3.3, there are facet unit normals $u_{0}, \ldots, u_{m}$ for the polytope $P_{j}$ that form a simplicial family, for some $m \geq d+1$. Since the facet normals of $P_{j}$ were taken from unit normals to regular points of $L$, this completes the proof.

Recall that a simplex $T$ circumscribes $L$ if $L \subseteq T$ and if $a T$ contains no translate of $L$ when $a<1$. An $n$-simplex $T \supseteq L$ circumscribes $L$ if and only if $L$ meets every facet of $T$. Theorem 3.4 therefore implies the following.

Corollary 3.5. Let $L \in \mathscr{K}_{n}$. Then $L$ is $(n-1)$-reliable if and only if there is no circumscribing $n$-simplex $T$ of $L$ such that $\partial T \cap L$ consists of regular points of $L$.

Since every boundary point of a smooth convex body is a regular boundary point, the following corollary is now immediate.

Corollary 3.6. If $L$ is a smooth convex body in $\mathbb{R}^{n}$, there exists an $n$-simplex $S$ such that $L_{u}$ contains a translate of $S_{u}$ for every unit direction $u$, while $L$ does not contain a translate of $S$.

We can now characterize 1-reliability.

Corollary 3.7. A convex set $L \in \mathscr{K}_{n}$ is a 1-reliable cover if and only if $L$ is a parallelotope.

Proof. If $L$ is a parallelotope then $L$ is 1-reliable, by Theorem 1.3 ,

Conversely, if $L$ is 1-reliable, then Theorem 3.4 asserts that there are no simplicial sets of size 3 or more among the unit normals at regular points of $L$. By Proposition 2.4 we may assume, without loss of generality, that $L$ has interior. In this case there exist affinely independent unit normals $u_{1}, \ldots, u_{m}$ at regular points of $L$, where $m \geq n+1$, and where the $u_{i}$ do not all lie in the same hemisphere. It follows that

$$
o=c_{1} u_{1}+\cdots+c_{m} u_{m}
$$

for some $c_{1}, \ldots, c_{m}>0$. By Proposition 2.2. the set of regular normals of $L$ has the form $\left\{ \pm v_{1}, \ldots, \pm v_{n}\right\}$, for some linearly independent set $v_{1}, \ldots, v_{n} \in \mathbb{S}^{n-1}$. Let $P$ be the unique (up to translation) parallelotope having facet unit normals $\pm v_{i}$ and corresponding facet areas $c_{i}$. Since the regular points of $L$ are dense in the boundary of $L$, it follows that $L$ and $P$ must be translates.

Corollary 3.8. Suppose that $P$ is a polytope in $\mathbb{R}^{n}$. Then $P$ is a d-reliable cover if and only if, for all $m \geq d+2$, no $m$ facets of $P$ share normal directions with an $(m-1)$-simplex. 
Since no four facet normals of the square pyramid $P$ in $\mathbb{R}^{3}$ contain the origin in the interior of their convex hull, any $K \in \mathscr{K}_{3}$ that can "hide behind" $P$ can also "hide inside" $P$. In other worlds, $P$ is 2-reliable, in spite of being indecomposable.

\section{Centrally symmetric Covering Sets}

We saw in the previous section that $L$ is a reliable 1-cover if and only if $L$ is 1-decomposable (i.e. a parallelotope). However, the square pyramid is 2-reliable in spite of being indecomposable.

A compact convex set $L$ is said to be centrally symmetric if $L$ and $-L$ are translates. For equivalence of 2-reliability and 2-decomposability to hold, we must restrict our attention to centrally symmetric bodies.

Theorem 4.1. A centrally symmetric set $L \in \mathscr{K}_{n}$ is 2-reliable if and only if $L$ is 2-decomposable.

The 3-dimensional case of Theorem 4.1 has the following especially simple form.

Corollary 4.2. A centrally symmetric set $L \in \mathscr{K}_{3}$ is 2-reliable if and only if $L$ is a cylinder.

The proof of Theorem 4.1 will use the following auxiliary results.

Proposition 4.3. Let $P$ be a convex polytope in $\mathbb{R}^{n}$ with non-empty interior. Suppose that $\xi$ is a proper subspace of $\mathbb{R}^{n}$, and suppose that each facet unit normal of $P$ lies either in $\xi$ or in $\xi^{\perp}$.

Then there exist polytopes $P_{1} \subseteq \xi$ and $P_{2} \subseteq \xi^{\perp}$ such that $P=P_{1} \oplus P_{2}$.

Proof. Suppose that the facet unit normals of $P$ are given by

$$
\left\{u_{1}, \ldots, u_{p}, v_{1}, \ldots, v_{q}\right\},
$$

where $u_{1}, \ldots, u_{p} \in \xi$ and $v_{1}, \ldots v_{q} \in \xi^{\perp}$. Suppose that each facet of $P$ with normal $u_{i}$ has area $a_{i}$ and each facet with normal $v_{j}$ has area $b_{j}$. By the Minkowski condition,

$$
a_{1} u_{1}+\cdots+a_{p} u_{p}+b_{1} v_{1}+\cdots+b_{q} v_{q}=o .
$$

It follows from the independence of $\xi$ and $\xi^{\perp}$ that

$$
a_{1} u_{1}+\cdots+a_{p} u_{p}=o \text { and } b_{1} v_{1}+\cdots+b_{q} v_{q}=o .
$$

By the Minkowski Existence Theorem [2, 28] there exists a polytope $Q_{1} \subseteq \xi$ having facet normals $u_{i}$ and corresponding facet areas $a_{i}$. Similarly, there exists a polytope $Q_{2} \subseteq \xi^{\perp}$ having facet normals $v_{j}$ and corresponding facet areas $b_{j}$.

Let $d=\operatorname{dim} \xi$, so that $\operatorname{dim} \xi^{\perp}=n-d$. For $x, y>0$, the Minkowski sum $x Q_{1}+y Q_{2}$ has the same unit normals as $P$ and has corresponding facets $x Q_{1}^{u_{i}}+$ $y Q_{2}$ and $x Q_{1}+y Q_{2}^{v_{j}}$, having the respective facet areas $x^{d-1} y^{n-d} V_{n-d}\left(Q_{2}\right) a_{i}$ and $x^{d} y^{n-d-1} V_{d}\left(Q_{1}\right) b_{j}$. Set

$$
x=\left(\frac{V_{n-d}\left(Q_{2}\right)^{n-d-1}}{V_{d}\left(Q_{1}\right)^{n-d}}\right)^{\frac{1}{n-1}} \quad \text { and } \quad y=\left(\frac{V_{d}\left(Q_{1}\right)^{d-1}}{V_{n-d}\left(Q_{2}\right)^{d}}\right)^{\frac{1}{n-1}}
$$

and let $P_{1}=x Q_{1}$ and $P_{2}=y Q_{2}$. The polytope $P_{1} \oplus P_{2}$ now has the same facet normals and the same corresponding facet areas as $P$. It follows from the uniqueness assertion of the Minkowski Existence Theorem that $P$ and $P_{1} \oplus P_{2}$ must be translates. 
Proposition 4.4. Let $K \in \mathscr{K}_{n}$ have non-empty interior. Suppose that there is a subspace decomposition $\mathbb{R}^{n}=\xi \oplus \xi^{\prime}$ such that each unit normal at a regular point of $K$ lies either in $\xi$ or in $\xi^{\prime}$.

Then there is a subspace decomposition $\mathbb{R}^{n}=\eta \oplus \eta^{\prime}$, where $\operatorname{dim} \eta=\operatorname{dim} \xi$ and $\operatorname{dim} \eta^{\prime}=\operatorname{dim} \xi^{\prime}$, and compact convex sets $K_{1} \subseteq \eta$ and $K_{2} \subseteq \eta^{\prime}$ such that $K=$ $K_{1} \oplus K_{2}$.

Proof. To begin, suppose that $\xi^{\prime}=\xi^{\perp}$, so that $\mathbb{R}^{n}=\xi \oplus \xi^{\prime}$ is an orthogonal decomposition. Since regular points are dense on the boundary of $K$ (see [28, p. 73]), there exists a countable dense set of regular points on the boundary of $K$. By intersecting half-spaces that support $K$ at these points, construct a sequence of polytopes $P_{i}$, decreasing with respect to set inclusion, such that $P_{i} \rightarrow K$ and each $P_{i}$ has facet normals that are unit normals at regular points of $K$.

By Proposition 4.3, each $P_{i}=Q_{i} \oplus Q_{i}^{\prime}$, where $Q_{i} \subseteq \xi$ and $Q_{i}^{\prime} \subseteq \xi^{\prime}$. Since projections are continuous, the $Q_{i}=\left(P_{i}\right)_{\xi}$ converge to $K_{\xi}$, and similarly $Q_{i}^{\prime} \rightarrow K_{\xi^{\prime}}$. Therefore $K=\lim _{i} P_{i}=K_{\xi} \oplus K_{\xi^{\prime}}$.

More generally, if $\xi$ and $\xi^{\prime}$ are not orthogonal complements, then let $\psi: \mathbb{R}^{n} \rightarrow$ $\mathbb{R}^{n}$ be a non-singular linear transformation such that $\psi^{-T} \xi \perp \psi^{-T} \xi^{\prime}$, where $\psi^{-T}$ denotes the inverse transpose of $\psi$. Let $\eta=\psi^{-1} \psi^{-T} \xi$ and $\eta^{\prime}=\psi^{-1} \psi^{-T} \xi^{\prime}$.

Recall that $(\psi \xi)^{\perp}=\psi^{-T}\left(\xi^{\perp}\right)$. Therefore, if each unit normal at a regular point of $K$ lies either in $\xi$ or in $\xi^{\prime}$, then each unit normal at a regular point of $\psi K$ lies either in $\psi^{-T} \xi$ or in $\psi^{-T} \xi^{\prime}$. Since these subspaces form an orthogonal decomposition, the previous argument implies that $\psi K=L_{1} \oplus L_{2}$, where $L_{1} \subseteq \psi^{-T} \xi$ and $L_{2} \subseteq \psi^{-T} \xi^{\prime}$. It follows that $K=K_{1} \oplus K_{2}$, where $K_{1}=\psi^{-1} L_{1} \subseteq \eta$ and $K_{2}=\psi^{-1} L_{2} \subseteq \eta^{\prime}$.

Proof of Theorem 4.1. If $L$ is 2-decomposable then $L$ is 2-reliable by Theorem 1.3 .

For the converse, suppose that $L$ is 2-reliable. Let $A$ denote the set of unit normals at regular points of $L$. Since $L$ is 2-reliable, $A$ contains no simplicial subsets sets of size 4 , by Theorem 3.4 .

Since $L$ is centrally symmetric, we have $A=-A$. By Proposition 2.3, there exists a subspace direct sum decomposition

$$
\mathbb{R}^{n}=W_{1} \oplus \cdots \oplus W_{k}
$$

where each $\operatorname{dim} W_{i} \leq 2$, and such that $A \subseteq W_{1} \cup \cdots \cup W_{k}$. It follows from Proposition 4.4 that $L$ is 2-decomposable.

In view of Theorem 4.1 one may be tempted to conjecture that $d$-reliability is equivalent to $d$-decomposability for centrally symmetric bodies, but this turns out to be false for $d=3$. Consider the following 12 vectors in $\mathbb{R}^{4}$ :

$$
\pm(1,1,0,0), \pm(1,0,1,0), \pm(1,0,0,1), \pm(0,1,1,0), \pm(0,1,0,1), \pm(0,0,1,1) .
$$

By Minkowski's Existence Theorem, there exists a unique 12-faceted polytope $Q$ in $\mathbb{R}^{4}$, centrally symmetric about the origin (i.e. $Q=-Q$ ), having facet normals parallel to the directions above, with each facet having unit 3-volume. One can verify that the set of vectors above contains no simplicial 5-family, so that $Q$ is 3-reliable by Corollary 3.8, A routine linear algebra computation (using Proposition 4.4) also verifies that $Q$ is not 3-decomposable. 


\section{Some Open QUeSTIONS}

There remain several fundamental open questions about convex bodies and projections, among them the following:

I. Under what symmetry (or other) conditions on $L \in \mathscr{K}_{n}$ is $d$-reliability equivalent to $d$-decomposability, for $d>2$ ?

A solution to Problem I would generalize Corollary 3.7 and Theorem 4.1. For example, what happens if we assume that $L$ is a zonoid?

Denote the $n$-dimensional (Euclidean) volume of $L \in \mathscr{K}_{n}$ by $V_{n}(L)$.

II. Let $K, L \in \mathscr{K}_{n}$ such that $V_{n}(L)>0$, and let $1 \leq d \leq n-1$. Suppose that $L_{\xi}$ contains a translate of $K_{\xi}$ for every $d$-subspace $\xi$ of $\mathbb{R}^{n}$.

What is the best upper bound for the ratio $\frac{V_{n}(K)}{V_{n}(L)}$ ?

Some partial answers to Problem II are offered in [16. There it is shown that if $K_{\xi}$ can be translated inside $L_{\xi}$ for all $d$-dimensional subspaces $\xi$, then $K$ has smaller volume than $L$ whenever $L$ can be approximated by Blaschke combinations of $d$ decomposable sets. However, there are cases in which $V_{n}(K)>V_{n}(L)$, in spite of the covering condition on shadows. For all $L \in \mathscr{K}_{n}$ it is also shown that, if $K_{u}$ can be translated inside $L_{u}$ for all unit directions $u$, then $V_{n}(K) \leq n V_{n}(L)$, where $n$ is the dimension of the ambient space for $K$ and $L$. However, I doubt this is the best possible bound.

III. Let $K, L \in \mathscr{K}_{n}$, and let $1 \leq d \leq n-1$. Suppose that, for each $d$-subspace $\xi$ of $\mathbb{R}^{n}$, the orthogonal projection $K_{\xi}$ of $K$ can be moved inside $L_{\xi}$ by some rigid motion (i.e. a combination of translations, rotations, and reflections). Under what simple (easy to state, easy to verify) additional conditions does it follow that $K$ can be moved inside $L$ by a rigid motion?

Problem III is an intuitive generalization of the questions addressed in this article. Indeed, each question can be re-phrased allowing for rotations (and reflections) as well as translations. However, the arguments presented so far rely on the observation that the set of translates of $K$ that fit inside $L$, that is, the set

$$
\left\{v \in \mathbb{R}^{n} \mid K+v \subseteq L\right\},
$$

is a compact convex set in $\mathbb{R}^{n}$. By contrast, the set of rigid motions of $K$ that fit inside $L$ will lie in a more complicated Lie group. For this reason (at least) the questions of covering via rigid motions may be more difficult to address than the case in which only translation is allowed.

\section{REFERENCES}

[1] K. Ball, Some remarks on the geometry of convex sets, Geometric Aspects of Functional Analysis (J. Lindenstrauss and V.D. Milman, eds.), Springer Lecture Notes in Mathematics, vol. 1317, Springer Verlag, Berlin, 1988. MR950983 (89h:52009)

[2] T. Bonnesen and W. Fenchel, Theorie der konvexen Körper, Chelsea, New York, USA, 1948.

[3] _ Theory of Convex Bodies, BCS Associates, Moscow, Idaho, 1987. MR920366 $(88 \mathrm{j}: 52001)$

[4] J. Bourgain, On the Busemann-Petty problem for perturbations of the ball, Geom. Funct. Anal. 1 (1991), 1-13. MR.1091609 (92c:52008)

[5] H. Busemann and C. M. Petty, Problems on convex bodies, Math. Scand. 4 (1956), 88-94. MR0084791(18:922b) 
[6] R. J. Gardner, Intersection bodies and the Busemann-Petty problem, Trans. Amer. Math. Soc. 342 (1994), 435-445. MR1201126 (94e:52008)

[7] _ A positive answer to the Busemann-Petty problem in three dimensions, Ann. Math. (2) 140 (1994), 435-447. MR.1298719 (95i:52005)

[8] , Geometric Tomography (2nd Ed.), Cambridge University Press, New York, 2006. MR2251886 (2007i:52010)

[9] R. J. Gardner, A. Koldobsky, and T. Schlumprecht, An analytic solution to the BusemannPetty problem on sections of convex bodies, Ann. Math. (2) 149 (1999), 691-703. MR 1689343 (2001b:52011)

[10] R. J. Gardner and A. Volčič, Convex bodies with similar projections, Proc. Amer. Math. Soc. 121 (1994), 563-568. MR1185262 (94h:52005)

[11] A. Giannopoulos, A note on a problem of H. Busemann and C. M. Petty concerning sections of symmetric convex bodies, Mathematika 37 (1990), 239-244. MR1099772 (92c:52009)

[12] H. Groemer, Ein Satz über konvexe Körper und deren Projektionen, Portugal. Math. 21 (1962), 41-43. MR0142055 (25:5449)

[13] H. Hadwiger, Gegenseitige Bedeckbarkeit zweier Eibereiche und Isoperimetrie, Vierteljschr. Naturforsch. Gesellsch. Zürich 86 (1941), 152-156. MR0007274(4:112c)

[14] _ Überdeckung ebener Bereiche durch Kreise und Quadrate, Comment. Math. Helv. 13 (1941), 195-200. MR0004995 (3:90f)

[15] _ Seitenrisse konvexer Körper und Homothetie, Elem. Math. 18 (1963), 97-98. MR0155232 (27:5168)

[16] D. Klain, Covering shadows with a smaller volume, Adv. Math. 224 (2010), 601-619. MR2609017 (2011d:52012)

[17] - Containment and inscribed simplices, Indiana Univ. Math. J. 59 (2010), 1231-1244.

[18] D. Klain and G.-C. Rota, Introduction to Geometric Probability, Cambridge University Press, New York, 1997. MR.1608265 (2001f:52009)

[19] A. Koldobsky, Intersection bodies, positive definite distributions, and the Busemann-Petty problem, Amer. J. Math. 120 (1998), 827-840. MR1637955(99i:52005)

[20] D. G. Larman and C. A. Rogers, The existence of a centrally symmetric convex body with central sections that are unexpectedly small, Mathematika 22 (1975), 164-175. MR 0390914 $(52: 11737)$

[21] E. Lutwak, Intersection bodies and dual mixed volumes, Adv. Math. 71 (1988), 232-261. MR963487 (90a:52023)

[22] _ Containment and circumscribing simplices, Discrete Comput. Geom. 19 (1998), 229-235. MR1600051 (99a:52009)

[23] A. Papadimitrakis, On the Busemann-Petty problem about convex centrally symmetric bodies in $\mathbb{R}^{n}$, Mathematika 39 (1992), 258-266. MR1203282 (94a:52019)

[24] C. M. Petty, Projection bodies, Proceedings, Coll. Convexity, Copenhagen, 1965, vol. Københavns Univ. Mat. Inst., 1967, pp. 234-241. MR0216369 (35:7203)

[25] C. A. Rogers, Sections and projections of convex bodies, Portugal. Math. 24 (1965), 99-103. MR0198344 (33:6502)

[26] L. A. Santaló, Integral Geometry and Geometric Probability, Addison-Wesley, Reading, MA, 1976. MR0433364 (55:6340)

[27] R. Schneider, Zur einem Problem von Shephard über die Projektionen konvexer Körper., Math Z. 101 (1967), 71-82. MR0218976 (36:2059)

[28] _ Convex Bodies: The Brunn-Minkowski Theory, Cambridge University Press, New York, 1993. MR1216521 (94d:52007)

[29] G. C. Shephard, Shadow systems of convex bodies, Israel J. Math. 2 (1964), 229-236. MR0179686 (31:3931)

[30] V. Soltan, Convex sets with homothetic projections, Beiträge Algebra Geom. 51 (2010), 237249. MR2650489 (2011c:52010)

[31] R. Webster, Convexity, Oxford University Press, New York, 1994. MR.1443208 (98h:52001)

[32] G. Zhang, Intersection bodies and the Busemann-Petty inequalities in $\mathbb{R}^{4}$, Ann. Math. (2) 140 (1994), no. 2, 331-346. MR1298716 (95i:52004)

[33] _ A positive solution to the Busemann-Petty problem in $\mathbb{R}^{4}$, Ann. Math. (2) 149 (1999), 535-543. MR.1689339(2001b:52010) 
[34] J. Zhou, The sufficient condition for a convex body to contain another in $\mathbb{R}^{4}$, Proc. Amer. Math. Soc. 121 (1994), 907-913. MR1184090 (94i:52007)

[35] _ Sufficient conditions for one domain to contain another in a space of constant curvature, Proc. Amer. Math. Soc. 126 (1998), 2797-2803. MR.1451838 (98k:52016)

Department of Mathematical Sciences, University of Massachusetts Lowell, LowELl, Massachusetts 01854

E-mail address: Daniel_Klain@uml.edu 